\title{
Control of Underactuated Driftless Systems Using Higher-Order Averaging Theory
}

\author{
Patricio A. Vela ${ }^{\dagger \ddagger}$, Joel W. Burdick ${ }^{\ddagger 1}$ \\ ${ }^{\dagger}$ Control and Dynamical Systems $\quad{ }^{\ddagger}$ Mechanical Engineering \\ California Institute of Technology, Mail Code 107-81, Pasadena, CA, 91125 \\ pvela@cds.caltech.edu, jwb@robotics.caltech.edu
}

\begin{abstract}
This papers applies a recently developed "generalized averaging theory" to construct stabilizing feedback control laws for underactuated driftless systems. These controls exponentialy stabilize in the average; the actual system may orbit around the average. Conditions for which the orbit collapses to the averaged trajectory are given. An example validates the theory, demonstrating its utility.
\end{abstract}

\section{Introduction}

A tremendous amount of research has gone into understanding controllability and determining conditions under which a system is controllable. There is still a gap, however, between the tests that determine controllablility and the actual feedback laws that realize control. This paper demonstrates how a recently developed "generalized averaging theory" [1] may be used in conjunction with controllability tests to realize feedback control for underactuated driftless systems. Our approach is an easily implementable and understandable strategy for designing exponentially stabilizing controllers for such systems. The results hold to general orders of Lie bracketing. The method does not use a homogeneous norm to demonstrate the exponential stabilization, does not require complicated coordinate expansions, the construction of Lyapunov functions, or the pre-existence of stabilizing controllers. The complexity of the nonlinear analysis grows with the order of Lie bracketing. Due to space constraints, only lower orders will be discussed. Extensions to higher order follow the same principles, but may be more involved computationally.

We seek to unite averaging theory and nonlinear control design. Our generalized averaging theory, which captures the dynamics of a system to arbitrary orders of approximation [1], has its roots in prior work on series expansions by Magnus, Chen, and Agračhev and Gamkrelidze $[2,3,4]$. Sussman and Kawski have analyzed series expansions with respect to controllability[5]. Some of the strongest results tying controllability analysis to control design is found in the work of Sussman and Liu $[6,7,8]$.

Moving from anaylsis to the design of stabilizing feedback laws is a challenge. Bullo $[9,10]$ has developed

\footnotetext{
${ }^{1}$ This work was supported in part by the NSF Engineering Research Center grant NSF9402726
}

series expansions for similar flows and their concomitant approximate inversion formulas in the case of simple mechanical systems and kinematic actuation.

The control of underactuated driftless systems has been widely studied. Generically, smooth state-feedback will not stabilize these systems [11], leading to the use of timevarying or non-smooth feedback techniques. In the timevarying control domain, the use of sinusoidal functions with appropriate phasing leads to motion in Lie bracket directions [12]. The analysis of open-loop response to sinusoidal inputs has been well studied [13]. Some have used averaging methods to understand the nonlinear response under time-periodic inputs $[8,14]$. The closed loop problem for time-varying controls is more difficult and fewer general solutions exist. In [15], a method to transform asymptotically stabilized homogeneous systems into an exponentially stabilizing homogeneous system is given. Stabilization is with regards to a homogeneous norm. In [16], a solution requiring the use of homogeneity is found for global or local stabilization, but the algorithm is computationally difficult and stabilization is with respect to a homogeneous norm. Alternative methods exist, however, these solutions are largely found in specific application domains $[17,18]$.

Section 2 reviews the key elements of the generalized averaging theory developed in the companion paper [1]. Section 3 applies this theory to the control of underactuated driftless systems. Section 4 demonstrates the utility of the approach via an example.

\section{A Generalized Averaging Theory}

The flow of the differential equation,

$$
\dot{x}=X(x, t ; \epsilon)=\epsilon \widehat{X}(x, t), \quad x(0)=x_{0},
$$

with $X$ smooth in $x$, and $T$-periodic, i.e., $X(x, t ; \epsilon)=$ $X(x, t+T ; \epsilon)$ can be analyzed by a non-linear version of Floquet theory. This approach represents the flow as the composition of a periodic flow and the evolution of an averaged vector field. These can be approximated to arbitrary order by appropriate series expansions.

Theorem 1 (Nonlinear Floquet Theorem) [1] Let $\Phi_{0, t}^{X}$ be the flow of the time-periodic differential equation (1). If the monodramy map has a logarithm, then the 
flow $\Phi_{0, t}^{X}$ can be represented as a composition of flows $\Phi_{0, t}^{X}=P(t) \circ \exp (Y t) \mathrm{Id}$, where $P$ is $T$-periodic, and $Y$ is an autonomous vector field.

The monodramy map is the flow of $X$ at time $T$, e.g., $\Phi_{0, T}^{X}$. It coincides with the flow of the average autonomous vector field, $Y$, at time $T$, e.g., $\exp (Y T)$.

Theorem 2 [1] If the monodramy map has a fixed point, the actual flow has a periodic orbit whose stability is determined by the stability of the monodramy map.

Corollary 1 [1] If the flow of system (1) has a fixed point $x^{*}$, as does the monodramy map, then stability of the actual flow may be determined using the monodramy map. A linearly (asymptotically) stable fixed point for the monodramy map implies a linearly (asymptotically) stable fixed point for the system (1).

Averaging theory seeks to find suitable approximations to the infinite series expansions given by $P(t)$ and $Y$. The approximations are given by truncations of the series expansions for $P(t)$ and $Y$; the $\mathrm{m}^{\text {th }}$-order truncation will be denoted by $\operatorname{Trunc}_{m}(\cdot)$; see [1] for the structure of these truncations. The theorems below relate the properties of the truncated series versions of these maps to the full expansions.

Theorem 3 [1] The $\mathrm{m}^{\text {th }}$-order truncation of the logarithm of the monodramy map gives an $(\mathrm{m}+1)^{\text {th }}$-order flow approximation for finite time, i.e., for time $O(1)$

$$
\exp (Y t)=\exp \left(\operatorname{Trunc}_{m}(Y) t\right)+O\left(\epsilon^{m+1}\right) .
$$

Theorem 4 [1] An $\mathrm{m}^{\text {th }}$-order truncation of the timeperiodic Floquet mapping is of order $(m+1)$-close to the time-periodic Floquet mapping on the time scale o(1).

$$
P(t)=\operatorname{Trunc}_{m}(P(t))+O\left(\epsilon^{m+1}\right)
$$

Proposition 1 [1] If the Floquet mapping has a timeindependent bias, i.e., $P(t)=\widetilde{P}(t) \circ P_{0}$, then a new av eraged vector field may be written $Z=\left(P_{0}\right)_{*} Y$.

The evolution of the Floquet solution becomes, $x(t)=$ $\widetilde{P}(t) \circ \exp (Z t)$, with $z_{0}=P_{0}^{-1}\left(x_{0}\right)$.

\section{Control of Kinematic Systems}

The standard form for an underactuated driftless affine control system is

$$
\dot{q}=Y_{a}(q) u^{a}(q, t)
$$

defined on a domain $D \subset \mathbb{R}^{n}$ for $a=1, \ldots, m<n$. For driftless affine control systems, small-time local controllability (see [19] for details) is based on the Lie Algebra Rank Condition (LARC),

$$
\operatorname{dim} \bar{\Delta}(q)=\operatorname{dim} T_{q} D, \quad \forall q \in D
$$

where $\bar{\Delta}$ is the involutive closure of the control vector field distribution, $\Delta \equiv \operatorname{span}\left\{Y_{\mathrm{a}}\right\}$.
Theorem 5 (Chow's Theorem) [19] The system (3) is small-time locally controllable if and only if the Lie Algebra Rank Condition holds.

We assume that this theorem is satisfied, and now focus on relating the terms in $\bar{\Delta}$ to control system design.

\subsection{Averaging Theory for Control}

We consider control inputs that combine state feedback and time-periodic terms; $u^{a}(x, t)=f^{a}(x)+v^{a}(t / \epsilon)$, with $v^{a}(\cdot) T$-periodic. Substituting these controls into (3) gives,

$$
\dot{q}=Y_{a}(q) f^{a}(q)+Y_{a}(q) v^{a}(t / \epsilon)
$$

The functions $v^{a}(t)$ are $T$-periodic functions (typically with zero average) and the functions $f^{a}(q)$ stabilize the directly controlled states. A transformation of time,

$$
\frac{\mathrm{d} q}{\mathrm{~d} \tau}=\epsilon Y_{a}(q) f^{a}(q)+\epsilon Y_{a}(q) v^{a}(\tau)
$$

takes (5) into a form compatible with averaging theory.

The averaged system vector fields will contain combinations of time integrals and Lie brackets. Since the periodic inputs act as coefficients to the input vector fields, and iterated Lie brackets are multi-linear, the integrals can be factored. These integral terms represent the net effect of the inputs on the Lie brackets terms. Define the following notation for the averaging coefficients:

$$
\mathrm{V}_{(n)}^{(a)}(t)=\int_{0}^{t} \int_{0}^{s_{n-1}} \cdots \int_{0}^{s_{2}} v^{a}\left(s_{1}\right) d s_{1} \cdots d s_{n-1} .
$$

The time-averaged terms are averaged coefficients. Cases of multiple upper and lower indices denote products of this type of integral. E.g., $V_{(1,1)}^{(a, b)}(t)$ has the form

$\mathrm{V}_{\{1,1)}^{(a, b)}(t)=\mathrm{V}_{(1)}^{(a)} \mathrm{V}_{(1)}^{(b)}=\left(\int_{0}^{t} v^{a}\left(s_{1}\right) d s_{1}\right)\left(\int_{0}^{t} v^{b}\left(s_{1}\right) d s_{1}\right)$

Additionally define the following, $\widetilde{V}_{(n)}^{(a)} \equiv \mathrm{V}_{(n)}^{(a)}-\overline{V_{(n)}^{(a)}}$ and for the multi-index version $\widetilde{\mathrm{V}}_{(N)}^{(A)} \equiv \mathrm{V}_{(N)}^{(A)}-\overline{\mathrm{V}_{(N)}^{(A)}}$ where $(A)=\left(a_{1}, a_{2}, \ldots, a_{|A|}\right)$ and $(N)=\left(n_{1}, n_{2}, \ldots, n_{|N|}\right)$. The $?$ symbol will denote integrals within the product structure. For example,

$$
\mathrm{V}_{(\widehat{0,0}, 1)}^{(\widehat{a, b}, c)}(t)=\left(\int_{0}^{t} \mathrm{~V}_{(0,0)}^{(a, b)}(\tau) \mathrm{d} \tau\right)\left(\mathrm{V}_{(1)}^{(c)}(t)\right)
$$

$1^{\text {st }}$ and $2^{\text {nd }}$-order averaging. The $1^{\text {st }}$ order averaged version of system (5) is,

$$
\dot{z}=Y_{\mathrm{a}}(z) f^{a}(z)+Y_{\mathrm{a}}(z) \overline{\mathrm{V}_{(0)}^{(a)}(t)} .
$$

Second order terms are typically used when the $1^{\text {st }}$-order average vanishes, or better approximations are desired. Assume that: $\overline{V_{(0)}^{(a)}(t)}=0$. All higher-order averages use 


$$
\begin{aligned}
\dot{z}=Y_{a} & (q) f^{a}(q)+\overline{\mathrm{V}_{(1)}^{(a)}(t)}\left[Y_{a}(z), Y_{b}(z) f^{b}(z)\right] \\
& +\frac{1}{2} \epsilon \overline{\mathrm{V}_{(1,0)}^{(a, b)}(t)}\left[Y_{a}(z), Y_{b}(z)\right] \\
& +\epsilon^{2}\left(-\frac{1}{2} T \overline{\mathrm{V}_{(1)}^{(c)}(t)}+\overline{\mathrm{V}_{(2)}^{(c)}}\right) \\
& +\epsilon^{2}\left(\frac{1}{2} \overline{\mathrm{V}_{(1,0)}^{(b, c)}(t)}+\frac{1}{3} T \overline{\mathrm{V}_{(1,0)}^{(b, c)}(t)}-\frac{1}{3} \overline{\mathrm{V}_{(\widehat{a}, b)}^{(\widehat{a, 0})}(t)}\right) \\
& +\frac{1}{3} \epsilon^{2}\left(\overline{\mathrm{V}_{(1,1)}^{(a, b)}(t)}-T \overline{\mathrm{V}_{(1,0)}^{(a, b)}(t)}+\overline{\mathrm{V}_{(\widehat{1,0)}}^{(\widehat{a, b)}}}\right) \\
& +\frac{1}{3} \epsilon^{2} \overline{\mathrm{V}_{(1,1,0)}^{(a, b, c)}(t)}\left[Y_{a}(z),\left[Y_{b}(z), Y_{c}(z)\right]\right]
\end{aligned}
$$

Table 1: $3^{\text {rd }}$-order average

this assumption. The $2^{\text {nd }}$ order averaged system has the form

$$
\begin{aligned}
\dot{z}=Y_{a}(z) f^{a}(z)+\epsilon \overline{\mathrm{V}_{(1)}^{(a)}(t)} & {\left[Y_{a}(z), Y_{b}(z) f^{a}(z)\right] } \\
+ & \frac{1}{2} \epsilon \overline{\mathrm{V}_{(1,0)}^{(a, b)}(t)}\left[Y_{a}(z), Y_{b}(z)\right]
\end{aligned}
$$

$3^{r d}$-order averaging If the LARC is satisfied via higher levels of iterated Lie brackets, then higher-order averaging is required. The averaged vector field, including third level iterated Lie brackets, is found in Table 1

The above analysis demonstrates that although Lie brackets determine possible flow directions, the averaged coefficients dictate the degree of flow in those directions. Since the LARC predicts the controllable directions, one would like to have a similar procedure to determine when input functions contribute to critical bracket directions.

\subsection{Sinusoidal Inputs for Indirect Actuation}

Ref.s [12] and [20] have demonstrated the use of sinusoidal inputs for motion generation in Lie bracket directions. By approximating the flow, one can compute the amplitudes of the sinusoidal functions for a given direction. This approximate inversion technique is successfully used in [10] and [21] to derive motion control algorithms for underactuated mechanical systems. We generalize this work and provide constructive control laws for underactuated driftless affine control systems.

Recent work on the $2^{\text {nd }}$-order averaged case has shown how to construct sinusoidal inputs with proper amplitude modulation and frequency spacing relations so as to isolate various Lie bracket contributions $[22,23]$. We now show how the averaged coefficients lead to these kinds of relations. Unless noted, the inputs are either cosines or sines with whole number frequency coefficients. $2^{\text {nd }}$-order Averaging Determining the inputs for arbitrary systems at any averaging order is difficult. However, guidelines can be established by investigating simple, lower-order cases. Abstraction to higher order follows naturally.

For $2^{\text {nd }}$-order averaging, the averaged coefficient is $\overline{\mathrm{V}_{(0,1)}^{(a, b)}}(t)$. The possible inputs for $v^{a}(t), a=1 \ldots m$, are $\alpha_{a} \sin \left(\omega_{a} t\right)$ or $\alpha_{a} \cos \left(\omega_{a} t\right)$. Of the four options for a desired input pair, simultaneous sinusoidal inputs do not work. Additionally, the algebraic equality

$$
\omega_{a}-\omega_{b}=0 .
$$

must hold for the desired input pair and for no other inputs. The net result will be a set of inputs that operate at unique carrier frequencies; a commonly known fact.

Lemma 1 For the case of two vector fields entering into the $2^{\text {nd }}$-order Lie brackets $\left[Y_{a}, Y_{b}\right]$, if the inputs

$$
v^{a}(t)=\alpha_{a b} \cos (\omega t), v^{b}(t)=\omega \sin (\omega t)
$$

are chosen for some principle carrier frequency, $\omega$, then only the bracket $\left[Y_{a}, Y_{b}\right]$ will be excited.

Higher Order Sinusoidal Actuation Higher-order expansions will have additional algebraic restrictions analogous to (8) in order to keep the effect of the inputs isolated. These restrictions will also affect the previous construction if both $2^{\text {nd }}$ and $3^{\text {rd }}$ order effects are desired simultaneously.

$3^{\text {rd }}$-order averaging Consider the third order averaged vector field with coefficient $\overrightarrow{\mathrm{V}_{(1,1,0)}^{(a, b, c)}(t)}$. The 8 possible input permutations lead to the contributions found in Table 2, with potential coupling found in Table 3. Thus, the important algebraic equalities are

$$
\omega_{1}+\omega_{2}-\omega_{3}=0, \omega_{1}-\omega_{2}-\omega_{3}=0, \omega_{1}-\omega_{2}+\omega_{3}=0 \text {. (10) }
$$

In order to avoid coupling between terms, the following inequalities may also need to hold,

$$
\omega_{i}-2 \omega_{j} \neq 0 \text {, or } \omega_{i} \neq \omega_{j} .
$$

With the above conditions met, the only non-zero combinations involve an odd number of cosines and an even number of sines.

This example highlights a few critical issues when moving to higher order. First, the coupling due to integrally related choices of frequency may fail to satisfy the algebraic inequalities (11). Secondly more than one Lie bracket may be simultaneously excited.

Lemma 2 When two distinct vector fields enter into the $3^{\text {rd }}$-order Lie brackets $\left[Y_{b},\left[Y_{a}, Y_{b}\right]\right]$, if the inputs

$$
v^{a}(t)=\omega \cos \left(2 \omega_{t}\right), v^{b}(t)=\alpha_{b a b} \omega \sin (\omega t)
$$

are chosen for some principle carrier frequency, $\omega$, then only the bracket $\left[Y_{b},\left[Y_{a}, Y_{b}\right]\right]$ will be excited. 


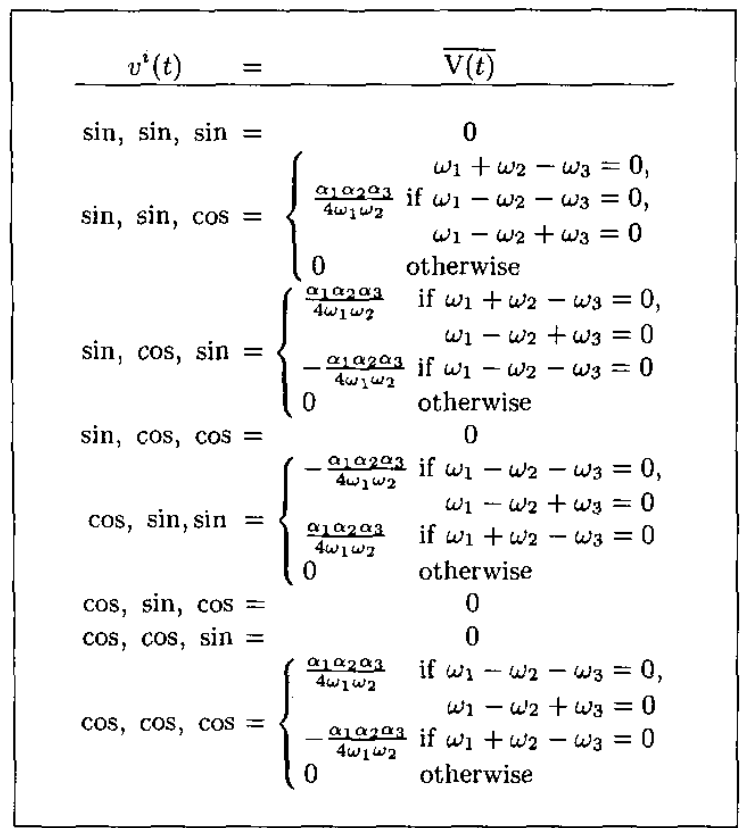

Table 2: Averaged coefficients for third order

\begin{tabular}{|cccc|}
\hline$v^{i}(t)$ & $\omega_{1}=\omega_{2}$ & $\omega_{1}=\omega_{3}$ & $\omega_{2}=\omega_{3}$ \\
\hline $\sin , \sin , \sin$ & 0 & 0 & 0 \\
$\sin , \sin , \cos$ & 0 & $-\frac{\alpha_{1} \alpha_{2} \alpha_{3}}{2 \omega_{1} \omega_{2}}$ & $-\frac{\alpha_{1} \alpha_{2} \alpha_{3}}{2 \omega_{1} \omega_{2}}$ \\
$\sin , \cos , \sin$ & 0 & 0 & $-\frac{\alpha_{1} \alpha_{2} \alpha_{3}}{2 \omega_{1} \omega_{2}}$ \\
$\sin , \cos , \cos$ & 0 & 0 & 0 \\
$\cos , \sin , \sin$ & 0 & $\frac{\alpha_{1} \alpha_{2} \alpha_{3}}{2 \omega_{1} \omega_{2}}$ & 0 \\
$\cos , \sin , \cos$ & 0 & 0 & 0 \\
$\cos , \cos , \sin$ & 0 & 0 & 0 \\
$\cos , \cos , \cos$ & 0 & 0 & 0 \\
\hline
\end{tabular}

Table 3: Coupling of averaged coefficients for third order

Proof: Assume for now that $v^{a}$ and $v^{b}$ are the only nonzero system inputs. Without loss of generality, let $a=1, b=2$. Set the input functions to be,

$$
v^{1}(t)=\alpha_{1} \cos (2 \omega t), v^{2}(t)=\alpha_{2} \sin (\omega t) .
$$

The critical elements of the averaged coefficient are,

$$
\widehat{V_{(1,1,0)}^{(c, d, c)}(t)}=\left[\begin{array}{cc}
{\left[\begin{array}{l}
0 \\
0
\end{array}\right]} & {\left[\begin{array}{c}
0 \\
-\frac{\alpha_{1} \alpha_{2}^{2}}{8 \omega^{2}}
\end{array}\right]} \\
{\left[\begin{array}{c}
0 \\
-\frac{\alpha_{1} \alpha_{2}^{2}}{8 \omega^{2}}
\end{array}\right]\left[\begin{array}{c}
\frac{\alpha_{1} \alpha_{2}^{2}}{4 \omega^{2}} \\
0
\end{array}\right]}
\end{array}\right],
$$

where the averaged coefficient is written like a tensor. With the choice of $\alpha_{1}=\alpha_{212}$ and $\alpha_{2}=\omega$,

$$
\overline{\mathrm{V}_{(1,1,0)}^{(c, d, c)}(t)}\left[Y_{a},\left[Y_{b}, Y_{a}\right]\right]=-\frac{3 \alpha_{212}}{8}\left[Y_{2},\left[Y_{1}, Y_{2}\right]\right],
$$

and, the sum over all averaged coefficients is,

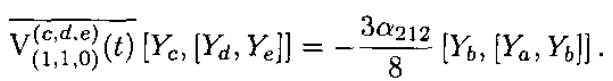

If there are other input functions, then $\omega$ must be chosen according to the algebraic equalities (10) and (11).

Lemma 3 When 3 distinct vector fields enter into a $3^{\text {rd }}$ order Lie bracket $(a \neq b, a \neq c$, and $b \neq c$ ), no choice of inputs results in a single bracket excitation. If the inputs

$$
\begin{gathered}
v^{a}(t)=\omega \cos (\omega t), v^{b}(t)=\omega \sin (3 \omega t) \\
v^{c}(t)=\alpha_{a b c} \sin (2 \omega t)
\end{gathered}
$$

are chosen for some principle carrier frequency, $\omega$, then only the bracket $\left[Y_{a},\left[Y_{b}, Y_{c}\right]\right]$ and one cyclicy related bracket, $\left[Y_{c},\left[Y_{a}, Y_{b}\right]\right]$ or $\left[Y_{b},\left[Y_{c}, Y_{a}\right]\right]$, will be excited.

Proof: Assume that these are the only nonzero system inputs. Without loss of generality, let $a=1, b=2$, and $c=3$, and also $\omega_{2}>\omega_{3}>\omega_{1}$, with the input functions,

$$
\begin{gathered}
v^{1}(t)=\alpha_{1} \cos \left(\omega_{1} t\right), v^{2}(t)=\alpha_{2} \sin \left(\omega_{2} t\right), \\
v^{3}(t)=\alpha_{3} \sin \left(\omega_{3} t\right) .
\end{gathered}
$$

The critical elements of the averaged coefficient tensor are:

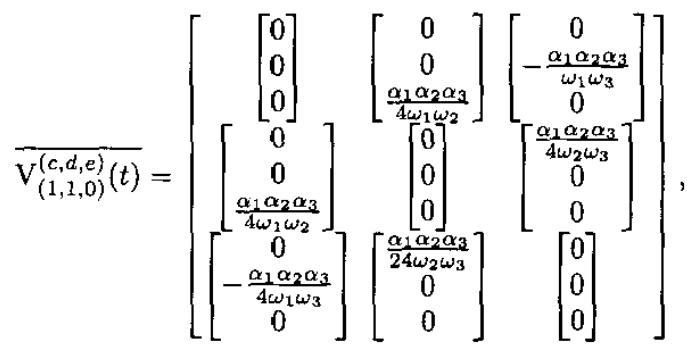

After grouping like Jacobi-Lie bracket terms and using the Jacobi-Lie identity,

$$
\begin{gathered}
\widehat{\mathrm{V}_{(1,1,0)}^{(c, d, e)}(t)}\left[Y_{c},\left[Y_{d}, Y_{e}\right]\right]=\frac{\alpha_{1} \alpha_{2} \alpha_{3}}{4}\left(\left(\hat{\omega}_{1}+\hat{\omega}_{3}\right)\left[Y_{1},\left[Y_{2}, Y_{3}\right]\right]\right. \\
\left.+\left(\hat{\omega}_{2}+\hat{\omega}_{3}\right)\left[Y_{2},\left[Y_{3}, Y_{1}\right]\right]\right)
\end{gathered}
$$

where $\hat{\omega}_{1}=\omega_{1}^{-1}\left(\omega_{2}^{-1}+\omega_{3}^{-1}\right), \hat{\omega}_{2}=\omega_{2}^{-1}\left(\omega_{3}^{-1}-\omega_{1}^{-1}\right)$, and $\hat{\omega}_{3}=\omega_{3}^{-1}\left(\omega_{1}^{-1}+\omega_{2}^{-1}\right)$. With these definitions,

$$
\hat{\omega}_{1}>0, \hat{\omega}_{2}<0, \hat{\omega}_{3}>0
$$

The second Lie bracket can be cancelled only if there exists a choice of $\omega_{i}$ satisfying one of the equalities (10), such that, $\hat{\omega}_{2}+\hat{\omega}_{3}=0$. This requires finding $\omega_{1}$ and $\omega_{3}$, acheiving the equality,

$$
\frac{2}{\left(\omega_{1}+\omega_{3}\right) \omega_{3}}-\frac{1}{\left(\omega_{1}+\omega_{3}\right) \omega_{1}}+\frac{1}{\omega_{1} \omega_{3}}=0,
$$

which is equivalent to $2 \omega_{1}-\omega_{3}+\left(\omega_{1}+\omega_{3}\right)=3 \omega_{1}=0$, an invalid solution. Thus, there is no choice of $\omega_{2}>\omega_{3}>\omega_{1}$ that leads to a single Lie bracket contribution.

As for the choice of inputs given above, notice that selecting any $\omega_{2}>\omega_{3}>\omega_{1}$ such that the equality $\omega_{2}=\omega_{1}+\omega_{3}$ holds will give a contribution with the two Lie brackets. Choosing $\omega_{3}=2 \omega_{1}=2 \omega$ will do the trick. Setting $\alpha_{1}=\omega, \alpha_{2}=\omega$, and $\alpha_{3}=\alpha_{123}$, the result is,

$$
\begin{aligned}
\overline{\mathrm{V}_{(1,1,0)}^{(a, b, c)}(t)} & {\left[Y_{a},\left[Y_{b}, Y_{c}\right]\right]=} \\
& \frac{\alpha_{123}}{8}\left(3\left[Y_{1},\left[Y_{3}, Y_{2}\right]\right]+\left[Y_{2},\left[Y_{3}, Y_{1}\right]\right]\right),
\end{aligned}
$$


Like the previous lemma, with other input functions present, $\omega$ must meet equalities (10) and (11).

This theorem is not as restrictive as it may seem. To excite only one of the terms, it will be necessary to expand the set of available input functions. Alternatively, one might have a vanishing bracket, e.g., $\left[Y_{b},\left[Y_{c}, Y_{a}\right]\right]=0$.

For higher order expansions, myriad algebraic identities may hold. Each averaged coefficient must be examined to determine its contribution, and the limitations arising from the chosen set of input functions. Note that once a particular calculation is done, it need not be repeated for another problem with the same Lie bracket structure.

\subsection{Stabilization Using Sinusoids}

To summarize, we have obtained the system response to an oscillatory control at some arbitrary order. We have also analyzed the effects of the control inputs on the expansions, leading to an $\alpha$-parametrized form. Now we must determine a stabilization feedback strategy.

For convenience, we introduce an ordering for the JacobiLie brackets. Listing the Jacobi-Lie brackets as they appear in the series expansion of the averaged vector field defines this ordering. Let $\widehat{\mathrm{Y}}_{j}$ denote the Jacobi-Lie brackets, and let $\mathrm{T}^{j}(\alpha)$ denote their corresponding averaged coefficients. With this ordering, the averaged equations can be put into the form:

$$
\begin{aligned}
\dot{z} & =Y_{a}(z) f^{a}(z)+\mathrm{T}^{j}(\alpha) \widehat{\mathrm{Y}}_{j}(z) \\
& =Y_{a}(x) f^{a}(x)+B(z) H(\alpha)
\end{aligned}
$$

where the matrices $B$ and $H$ are,

$$
B(z)=\left[\widehat{\mathrm{Y}}_{1} \ldots \widehat{\mathrm{Y}}_{N}\right] \text { and } H(\alpha)=\left[\mathrm{T}^{1} \ldots \mathrm{T}^{N}\right]
$$

Oscillatory Control via Discretized Feedback We use state error as feedback to modulate the parameters $\alpha$, converting the problem to periodic discrete feedback. It is very similar to motion control algorithms that use approximate inversion for open loop trajectory tracking.

Theorem 6 Consider a system (5) which satisfies the $L A R C$. Let $u^{k}(t)$ be the set of $\alpha$ parametrized, T-periodic input functions where $k=\{1, \ldots, m\}$ and $\alpha \in \mathbb{R}^{n-m}$. Let $z(t)$, be the averaged system response to the inputs. Given the averaged system (20), assuming that the $m$ directly controlled states have been linearly stabilized and that the linearization of $H$ with respect to $\alpha$ at $\alpha=0$ and $z=z^{*}$ is invertible on the $(n-m)$ dimensional subspace to control, there exists a $K \in \mathbb{R}^{(n-m) \times n}$ such that for

$$
\alpha=-\Lambda K z(T\lfloor t / T\rfloor)
$$

where $\Lambda^{(n-m) \times(n-m)}$ is invertible and $|\cdot|$ denotes the floor function, the average system response is stabilized.

Proof: The proof was essentially given in [23], but will be quickly sketched. Given the assumptions on the system, the averaged system (20) is controllable. Linearization with respect to $z$ and $\alpha$ yields

$$
\dot{z}=A z+B \frac{\partial H}{\partial \alpha} \alpha=A z+B \Gamma \alpha
$$

Choosing $\alpha$ constant over a period, the above system can be directly integrated to obtain a discrete, linear system

$$
z(k+1)=\hat{A} z(k)+\hat{B} \alpha .
$$

The assumptions imply that $\hat{B}$ has a pseudo-inverse, $\Lambda$, for the $(n-m)$-dimensional subspace to stabilize. Choose $K$ so that the eigenvalues of $\hat{A}-K$ lie in the unit circle. This stabilizes the discrete system, and the continuous system with piecewise constant feedback.

Comments. This theorems stabilizes an equilibrium point of the averaged system. To track a trajectory, replace $x(t)$ with $x(t)-x_{d}(t)$; the system must be locally controllable along the trajectory. If the $\alpha$-parametrized control input functions do not vanish at the equilibrium, then by Theorem 2, the flow of the actual system stabilizes to an orbit (of size $\epsilon$ ) around the fixed point. If, on the other hand, the input functions do vanish at the equilibrium, then Corollary 1 implies that the flow of the actual system stabilizes to the fixed point (i.e. the orbit collapses to the fixed point). Due to discretization of the feedback, the Nyquist criteria is a limiting factor in tracking a trajectory for the indirectly controlled states.

\section{Example}

The well-known nonholonomic integrator in $\mathbb{R}^{3}$ is a driftless system with two control inputs given by,

$$
Y_{1}(x)=\left[1,0,-x_{2}\right]^{T} \text { and } Y_{2}(x)=\left[0,1, x_{1}\right]^{T}
$$

The system is not linearly controllable, but does satisfy the LARC. Only second order averaging will be necessary. By the discussion in Section 3.2, out of phase sinusoids will excite the critical averaged coefficient. Define,

$$
v^{1}(t)=\alpha \sin (t), v^{2}(t)=\cos (t),
$$

and use state-feedback to stabilize the first two states, $f^{a}(x)=-3 x^{a}$. This choice of control inputs results in the averaged vector field, after linearization,

$$
\frac{\mathrm{d}}{\mathrm{d} t}\left\{\begin{array}{l}
z_{1} \\
z_{2} \\
z_{3}
\end{array}\right\}=\left\{\begin{array}{c}
-3 z_{1} \\
-3 z_{2} \\
0
\end{array}\right\}+\epsilon a\left\{\begin{array}{l}
0 \\
0 \\
1
\end{array}\right\},
$$

and can be stabilized according to Theorem 6 . The range of feedback gains that will stabilize the system can be derived as follows. Integrating over one period gives the following discrete time system:

$$
\begin{gathered}
z_{1}(k+1)=\exp (-3 T) z_{1}(k), z_{2}(k+1)=\exp (-3 T) z_{2}(k) \\
z_{3}(k+1)=z_{3}(k)+\epsilon T \alpha
\end{gathered}
$$

The first two states are exponentially stabilized (in the average). For $\alpha=-K z_{3}(k), K>0$, exponential stabilization occurs for $|1+\epsilon T K|<1$. The range of stabilizing feedback gains, $K \in\left(0, \frac{1}{\pi \epsilon^{2}}\right)$, depends on $\epsilon$. The most effective gain lies at the midpoint of the range, whereas the boundary coincides with nominal stability. Simulations with gains set outside of the boundary do diverge. The 


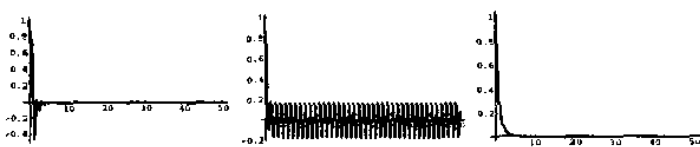

Figure 1: Orbit Stabilization for (22)

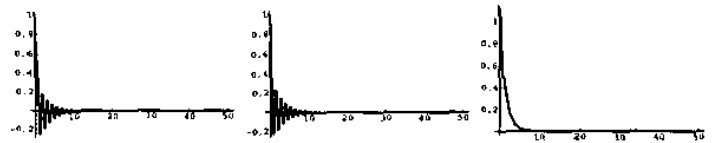

Figure 2: Point Stabilization for (24)

step response is plotted in Fig. 1 for $\epsilon=1 / 5$ and $K=3$. The averaged system response stabilizes exponentially, whereas the true system orbits about the origin. A different selection of the $\alpha_{a b}$ gives the inputs below,

$$
v^{1}(t)=\operatorname{sign}(\alpha) \sqrt{|\alpha|} \sin (t), v^{2}(t)=\sqrt{|\alpha|} \cos (t),
$$

and will exponentially stabilize the system via Corollary 1. The theory still applies, in spite of the periodic discontinuity in $v^{2}(t)$, and gives the same averaged vector field as equation (23). The response envelope has the characteristic exponent of the slowest converging mode.

\section{Conclusion}

A generalized averaging theory was applied to underactuated driftless affine control systems, resulting in an exponentially stabilizing control strategy. Since the control strategy and stabilization theorem are constructive, any system satisfying the conditions can be stabilized. This approach may have other attractive features. Since integration of the time dependent portions of the inputs smoothes out discontinuities, this approach can be used for legged locomotion control design. Recent research [24] studies this idea for a simple bipedal model. AIthough there are unique problems inherent to systems with drift, the general strategy set forth in this paper still holds in this case. For example, averaging methods have recently been applied to the stabilization of a carangiform fish [23] and the snakeboard [24]. After averaging, both of these systems with drift have remarkably similar control laws. Finally, the feedback stabilization proof used a discretized linear model that can be adapted to perform robust control under parametric uncertainty.

\section{References}

[1] Vela P.A. and Burdick J.W. A general averaging theory via series expansions. Proc. Amer. Contr. Conf., 2002.

[2] Magnus W. On the exponential solutions of differential equations for a linear operator. Comm. Pure Appl. Math., VII:649-673, 1954.

[3] Chen K. Integration of paths, geometric invariants and a generalized baker-hausdorff formula. The Annals of Mathematics, 65(1):163-178, 1957.

[4] Agračhev A. and Gamkrelidze R. The exponential representation of flows and the chronological calculus. USSR Sbornik, 35(6):727-785, 1978.

[5] Kawski M. and Sussman H. Noncommutative power series and formal lie-algebraic techniques in in nonlinear control theory. In D. Pratzel-Wolters U. Helmke and E. Zerz, editors, Operators, Systems, and Linear Algebra, pages 111128. Teubner, 1997.

[6] Lafferriere G. and Sussman H.J. Motion planning for controllable systems without drift. Proc. IEEE Int. Conf. Rob. Aut., pages 1148-53, 1991.

[7] Sussman H.J. and Liu W.S. Limits of highly oscillatory controls and the approximation of general paths by admissible trajectories. IEEE Conf. Dec. Contr., pages 437-442, 1991.

[8] Liu W.S. Averaging theorems for highly oscillatory differential equations and iterated lie brackets. SIAM J. Contr. Opt., 35(6):1989-2020, 1997.

[9] Bullo F. Averaging and vibrational control of mechanical systems. SIAM J. Contr. Opt., 41(2):542-62, 2002.

[10] Bullo F. Series expansions for the evolution of mechanical control systems. SIAM J. Contr. Opt., 40 (1):166-190, 2001.

[11] Brockett R.W. Asymptotic stability and feedback stabilization. In Differential Geometric Control Theory, pages 181-91. Birkhauser, 1993.

[12] Murray R.M. and Sastry S.S. Nonholonommic motion planning: Steering using sinusoids. IEEE Trans. Aut. Contr., 38(5):700-716, 1993.

[13] Brocket R.W. and Dai L. Nonholonomic kinematics and the role of elliptice functions in constructive controllability. In Progress in Nonholonomic Motion Planning, pages 1-22. Kluwer, 1992.

[14] Leonard N.E. and Krishnaprasad P.S. High-order averaging on lie groups and control of an autonomous underwater vehicle. UMD T.R. 93-70, 1993.

[15] M'Closkey R.T. and Murray R.M. Exponential stabilization of driftless nonlinear control systems using homogeneous feedback. IEEE Trans. Aut. Contr., 42(5):614-28, 1997.

[16] Morin P., Pomet J.-B., and Samson C. Design of homogeneous time-varying stabilizing control laws for driftless controllable systems via oscillatory approximation of lie brackets in closed loop. SIAM J. Contr. Opt., 38(1):22-49, 1999.

[17] Canudas de Wait C. and Sordalen O.J. Exponential stabilization of modile robots with nonholonomic constraints. IEEE Trans. Aut. Contr., 37(11):1791-7, 1992.

[18] Sordalen O.J. and Egeland O. Exponential stabilization of nonholonomic chanied systems. IEEE Trans. Aut. Contr., 40(1):35-49, 1995.

[19] Sastry S.S. Nonlinear Systems: Analysis, Stability, and Control. Springer, 1999.

[20] Teel A.R., Murray R.M., and Walsh G.C. Nonholonomic control systems: From steering to stabilization with sinusoids. Int. J. Cont., 62(4):849-870, 1995.

(21) Martínez S. and Cortés J. Motion control algorithms for simple mechanical systems with symmetry. submitted to Acta Applicandae Mathematicae, 2001.

[22] Vela P.A., Morgansen K.A., and Burdick J.W. Second order averaging methods for oscillatory control of underactuated mechanical systems. Proc. Amer. Contr. Conf., 2002.

[23] Vela P.A., Morgansen K.A., and Burdick J.W. Underwater locomotion from oscillatory shape deformations. IEEE Conf. Dec. Contr., 2002.

[24] Vela P.A. and Burdick J.W. Control of underactuated mechanical systems via averaging theory. to appear IEEE Int. Conf. Rob. Aut., 2002. 